\title{
Physical quantity as a pseudo-euclidean vector
}

\author{
Valery Mazin \\ St. Petersburg State Polytechnical University, UI. Polytechnicheskaja, 29, 195251 St. Petersburg, Russia
}

\section{ABSTRACT}

The aim of this research was an attempt of the finding of a mathematical model for a physical quantity organically including its degree of uncertainty. The basic method is an application of the geometrical approach. As the starting point the basic equation of measurement is accepted. Result was a presentation of a physical quantity by a vector of the pseudo-Euclidean plane. Four new matters appearing by such presentation are discussed.

\section{Section: RESEARCH PAPER}

Keywords: pseudo-euclidean plane; uncertainty; vector of the unit; total vector; pseudo-euclidean angle

Citation: Valery Mazin, Physical quantity as a pseudo-euclidean vector, Acta IMEKO, vol. 4, no. 4, article 3, December 2015, identifier: IMEKO-ACTA-04 (2015)-04-03

Section Editor: Franco Pavese, Torino, Italy

Received April 8, 2015; In final form June 17, 2015; Published December 2015

Copyright: (C) 2015 IMEKO. This is an open-access article distributed under the terms of the Creative Commons Attribution 3.0 License, which permits unrestricted use, distribution, and reproduction in any medium, provided the original author and source are credited

Corresponding author Valery Mazin, e-mail: masin@list.ru

\section{INTRODUCTION}

The categories of evolution in theoretical metrology, mentioned in [1], among which developing and substantiating mathematical models should be particularly noted, are still holding their truth today.

The geometrical approach plays a special role among other approaches to the understanding of fundamental categories of measurement. In the "Encyclopedia of Mathematics" [2], the role of Geometry is defined as follows: "The development of geometry, its applications, development of the geometric perception of abstract objects in different areas of mathematics and natural science give evidence of the importance of geometry as one of most drastic and productive means of reality cognition in terms of produced ideas and methods".

It is underscored in [3], that the geometric representation of analytic concepts has an infinite heuristic value; it also mentions that geometry "becomes more and more important in ... physics, simplifying mathematical formalities and deepening physical comprehension.

This renaissance of geometry influenced not just special and general theories of relativity, known to be geometrical in their essence, but also other branches of physics, where geometry of physical space is being replaced by the geometry of more abstract spaces".

This article offers for consideration a geometrical representation of a fundamental concept in metrology - the physical quantity. Also, a geometric representation integrates uncertainty into the concept of the physical quantity.

\section{DEFINING THE PROBLEM}

Let a quantity $x$ characterize an object, that allows it to change from $x_{a}$ to $x_{b}$. This very situation actually takes place in reality, for not a single physical parameter in an imperfect world can be defined by a single value. There is always a probable turn-down range, that exists either due to transition from one kind to another, or due to external conditions change, or due to inaccuracy of a measurement.

The mean value and the range of uncertainty characterizes different properties of a quantity (one is indicating the location on the numerical axis, the other - dispersion around this location). On the other hand, a physical quantity contains both, quantitative as well as qualitative aspects.

Both of these dualities suggest an idea that a representation of a quantity, naturally combining this duality, would be necessary.

This research attempts to represent any physical quantity as a two-dimensional vector of a given abstract space.

\section{PHYSICAL QUANTITY AS A VECTOR, CHARACTERIZING ITS UNCERTAINTY}

The following suggestion was proven in [4]: 
A collection of logarithms of physical quantities in any power (integer and fractional) plus Zero defines a Vector Space.

Each vector of this vector space, per basic equation of measurement $x=\{x\}[x]$, where $x$ is a physical quantity, $\{x\}$ is its numerical value, $[\mathrm{x}]$ is its unit, can be decomposed into two portions, formed up after taking the logarithm of this equation (strictly speaking, this simple notion is, of course, not an equation):

$\ln x=\ln \{x\}+\ln [x]$.

Expression (1) is usually considered lacking mathematical rigor and leading to contradictory statements, for it is commonly adopted that transcendent functions of denominate quantities do not make sense, whereas the units of quantities are exactly what make them as such.

Nevertheless, formal operation (1), obviously, can be carried out, and we will show that they are not just formal.

We shall distinguish between the fundamental possibility of assigning a coordinate system and the method of determining the values of these coordinates.

Taking the logarithm produces a coordinate system.

As for the values of coordinates, multiple approaches are possible. First of all, it is necessary to acknowledge that for as long as the unit of a quantity may have different values, this unit in itself bears a quantitative meaning. This meaning, however, remains indeterminate, for the entire quantitative side of a physical quantity is a result of comparing this quantity with unity.

Therefore logarithmic transformation can be used.

Secondly, as it will be shown later, by using the immutable fact of uncertainty of value of a quantity, it will become possible to make sense of $\ln [\mathrm{x}]$.

And finally, we can consider $\ln [\mathrm{x}]$ to be simply a symbol of a coordinate, a sign of direction, whereas $\alpha$ i shall be considered the coordinate itself.

Assuming $\sqrt{x_{a} x_{b}}=x_{\text {med }}$, corresponding vectors may be drawn as illustrated in Figure 1, where $a_{0}$ is the axis of numerical values logarithms. We will choose the metric on this plane in such a way, that it reflects the fact of the presence of $x$ in given limits. This can be done on the condition that the vectors magnitudes within their domain of definition were real, and outside the domain of definition - imaginary.

As a result, we shall arrive to:

$$
\left|\ln x_{a}\right|=\left|\ln x_{b}\right|=0 \text {, }
$$

i.e. corresponding vectors turn out to be isotropic.

All vectors, located to the left as well as to the right from the isotropic ones, including those, directed alongside the numerical axis, have imaginary lengths. The latter statement meets our condition, whose meaning is clear: what falls short of reality - is imaginary.

As abstract numbers don't exist in nature, a numerical axis must be imaginary. Such metric happens to be pseudoEuclidean.

Every point A of this plane characterizes an equivalent-kind physical quantity, taken in any power and varying within certain limits. We shall name it a representing point. In that case, the meaning of the points population, forming a pseudo-Euclidean plane, becomes clear if one can imagine few possible reasons for transitioning from one point to another, i.e. movements in this plane. These reasons are mathematically expressed by parameters, whose variations cause the end of the vector of

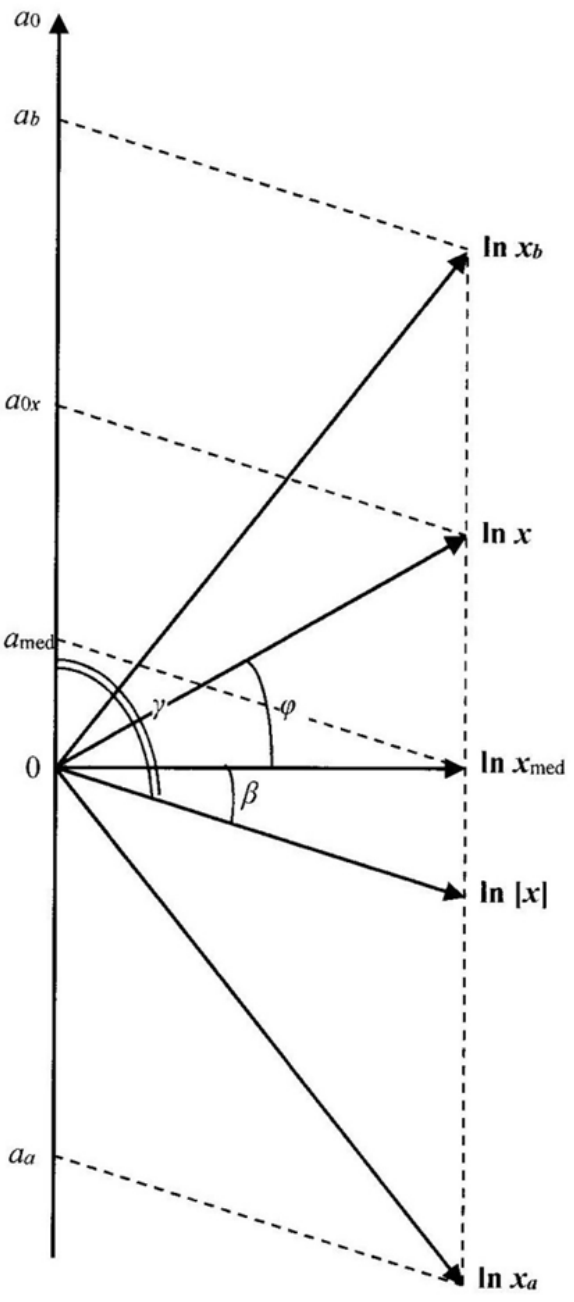

Figure 1. Vector representation of a physical quantity.

quantity trace a certain trajectory. It is possible to specify three types of such parameters: coverage probability, power of the quantity and external factors.

In such metric

$|\ln x|=\sqrt{\left|\ln x_{\text {med }}\right|^{2}-\left(a_{0 x}-a_{\text {med }}\right)^{2}}$.

This leads to

$\left|\ln \boldsymbol{x}_{\mathrm{med}}\right|=a_{b}-a_{\mathrm{med}}=a_{\mathrm{med}}-a_{a}$,

and

$|\ln [x]|=\sqrt{\left|\ln x_{\text {med }}\right|^{2}-a_{\text {med }}^{2}}$.

Substituting (4) into (5) and taking into consideration that

$a_{\mathrm{med}}=\frac{a_{a}+a_{b}}{2}$,

we obtain:

$\mid \ln [x]=\sqrt{-a_{a} a_{b}}$.

This result is of fundamental importance. It shows that the vector norm, representing the unit of a quantity, is not a permanently set value, but rather is a geometric average of logarithms of the limit values for a given quantity. After simple transformations we also arrive to:

$|\ln x|=\sqrt{\left(a_{b}-a_{0 x}\right)\left(a_{0 x}-a_{a}\right)}$. 
As a result of this concept, new entities are coming to life. The first of them - vector norm $\ln [\boldsymbol{x}]$.

\section{VECTOR OF A UNIT}

The vector norm of a unit generally is not equal to Zero since it is not a "logarithm of one" in its common sense, but rather is thought to be a logarithm of a certain qualitative content of the quantity. This notion fully agrees with the concept, stating that the numerical value and unit for a quantity reflect its quantitative and qualitative component, respectively.

Being expressed as a vector, laid in a pseudo-Euclidean plane, the unit of quantity thus has two dimensions. One of them is ordinary, with logarithmic coordinate at 0 , characterizes the size of the commonly adopted unit. Another dimension also gives the size of the unit, except this unit is related to the applicable physical system, and it is defined by the range $x_{a}, x_{b}$.

\section{THE FULL VECTOR OF A QUANTITY}

Another new entity, appearing in connection with the geometrical concept of a physical quantity - the length of its full vector $|\ln \boldsymbol{x}|$. As it can be seen from (6), when either $a_{0 x}=a_{a}$, or $a_{0 x}=a_{b},|\ln \boldsymbol{x}|=0$.

It can be explained only on the condition that $a_{0 \mathrm{x}}$ is located at the center of a corresponding probability distribution. In this case, if the center of distribution coincides with its limit, dispersion becomes Zero (for there aren't any values at either side from the center); then $a_{0 \mathrm{x}}$ becomes constant. No other interpretation is capable to give an informatively sound explanation of this exceptional nature of limit values for $a_{0 \mathrm{x}}$.

Moreover, we can also determine the class of distribution center for a quantity, represented by the coordinate $a_{0 \mathrm{x}}$ in a given model.

Since the numerical axis is "common for everyone", i.e. all values for distribution centers for all physical quantities are being located along it, these centers shall be added up algebraically while the summation itself is a vector addition.

It is worth to mention here, that this rule of addition is applicable in case of dealing with population means.

Consequently, $a_{0}$ is the population mean of logarithms of quantity $x$.

It is necessary to notice, that in each and every single pseudo-Euclidean plane, characterizing a certain quantity, any of known characteristics of the probability distribution center may act as coordinate $a_{0 x}$.

Having said that, if the quantities are multiplied (which is the same as vector sum of their logarithms), then a rule that governs addition of their logarithmic probability distribution centers shall be defined.

Likely, this will require establishing different numerical axes for different quantities. In this article, however, we will skip this issue.

Let's notice that the reason for a physical quantity to be represented as a two-dimensional entity lays in the fundamental uncertainty of its value. Such uncertainty causes the averages of $a_{0}$ and $|\ln [\boldsymbol{x}]|$ to have different absolute values.

The length of the quantity vector expresses the degree of its uncertainty in a format of geometric average of the two greatest possible deviations of quantity value logarithms from their center of distribution.

Let's re-write (8) in the following format: $|\ln x|=\sqrt{\ln \frac{\left\{x_{b}\right\}}{\{x\}} \cdot \ln \frac{\{x\}}{\left\{x_{a}\right\}}}=\sqrt{\ln \frac{x_{b}}{x} \cdot \ln \frac{x}{x_{a}}}$,

where $x=\exp a_{0 x}$. If the quantity's variation within the limits $x_{a}-x_{b}$ is reasonably small (owing to say, measurement errors), then the quotients $\frac{x_{b}}{x}$ and $\frac{x}{x_{a}}$ are approaching 1 .

Then, expanding the logarithms into Taylor series and limit the expansion to the first two members, we arrive at:

$|\ln x| \cong \sqrt{\left(\frac{x_{b}}{x}-1\right)\left(\frac{x}{x_{a}}-1\right)}=\sqrt{-\gamma_{+} \gamma_{-}}$,

where $\gamma_{+}$and $\gamma_{-}$- are positive and negative estimates of the error, respectively. Quite often $\gamma_{+}=\gamma_{-}=\gamma$ [5], consequently

$|\ln x| \equiv \gamma$.

Quantity variation limits of $x_{a}$ and $x_{b}$ define a level of confidence, specified with a certain probability. Consequently, the entire model built as a pseudo-Euclidean plane turns out to be probabilistic.

\section{THE ANGULAR CHARACTERISTIC OF MEASURABLE PROPERTY OF A PHYSICAL OBJECT}

The third new entity that needs much thinking, is the pseudo-Euclidean angle between the vector of quantity's unit and the full vector of the quantity. See Figure 2. Each point on a straight line, coinciding with the vector of quantity $x$, including the point, that belongs to $x$ in a fractional power, expresses the same property of the object in both, qualitative and quantitative, terms. For example, electric resistance $\mathrm{R}, \mathrm{R}^{2}$, conductivity $\mathrm{R}^{-1}$, and so on express the same property of a conductor, though in various terms. But then vector $\ln \boldsymbol{x}$, as well as any other vector co-linear to it, such as $\ln \boldsymbol{x}^{2}$, characterizes just one image of this general property. It is natural to assume, that there should be a characteristic that unites all of these images. This characteristic, obviously, is the angle at which the line $u$ crosses some chosen direction; for example, the direction of the quantity's unit vector, as shown in Figure 2. The angle between these straight lines, just as the quantity $x$ itself, characterizes the object in the adopted system of measurement; however, more general and more sustainable. It is certainly not the angle that can be seen, but rather a pseudo-Euclidean angle. Thus, we are forced to consider the angle as a characteristic of a measurable physical property of an object.

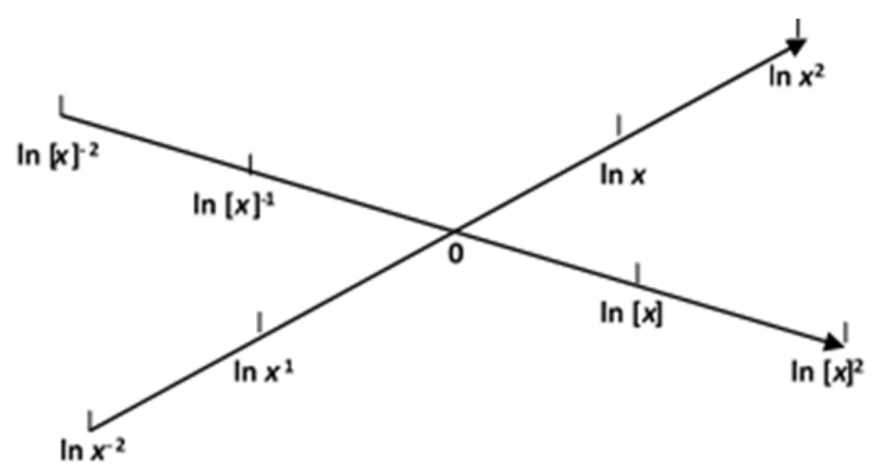

Figure 2. Further elaboration of the physical quantity concept. 
For the purpose of making the notes look simpler, we shall write: $\ln \boldsymbol{x}=x^{*} ; \ln [\boldsymbol{x}]=[x]^{*} ;\left|x_{\text {med }}^{*}\right|=l_{\text {med }} ; \frac{\left|[x]^{*}\right|}{|\mathbf{e}|}=a_{x}$, where $\boldsymbol{e}$ is a unitary vector, directed along $[x]^{*}$.

To begin with, let's define the angle $\theta_{\beta}$ between the unitary vector and the geometric average vector. Corresponding transformations show:

$\theta_{\beta}=\frac{1}{2} \ln \left(-\frac{a_{b}}{a_{a}}\right)$.

In other words, the pseudo-Euclidean angle between the unitary vector and the geometric average vector for this object is determined by the logarithm of ratio of quantity's limit values.

Formula (7) establishes the degree of difference between $\left|[x]^{*}\right|$ and $l_{\text {med }}$ and it is significant in the sense that $x_{\text {med }}$ can also be considered a system unit.

Now let's define the angle $\theta_{\varphi}$ between the vector of geometric average and the full vector of the quantity $x$, an angle shown in Figure 1 as $\varphi$. We find:

$\theta_{\varphi}=\frac{1}{2} \ln \frac{a_{0 x}-a_{a}}{a_{b}-a_{0 x}}$.

In this case, the logarithm is taken from a prime relation, that includes three points $-a_{a}, a_{0}, a_{b}$-all lying on the same line, a. k. a. an affine invariant.

Finally, the formula for the angle between the vector of the unit and the full vector of the quantity is expressed as

$\theta=\theta_{\beta}+\theta_{\varphi}=\frac{1}{2} \ln \left(-\frac{a_{b}}{a_{a}} \cdot \frac{a_{0 x}-a_{a}}{a_{b}-a_{0 x}}\right)=\frac{1}{2} \ln \left(\frac{a_{0 x}-a_{a}}{a_{b}-a_{0 x}}: \frac{0-a_{a}}{a_{b}-0}\right)$.

Here the logarithm is taken from a complex relation of four points - $a_{a}, a_{b}, a_{0_{x}}$, and 0 , representing a principal projective invariant. It is remarkable that the last formula exactly matches the formula defining the length $0-a_{0 x}$ in hyperbolic and elliptic geometries. It is analogous to the Laguerre formula

$\psi=\frac{i}{2} \ln \left(j_{1} j_{2} u_{1} u_{2}\right)$,

where $\psi$ is the Euclidean angle in a complex Euclidean space; $u_{1}$ and $u_{2}$ are two real straight lines; $j_{1}$ and $j_{2}$ are two isotropic imaginary straight lines, whereas all four straight lines pass through the same point; $\left(j_{1} j_{2} u_{1} u_{2}\right)$ is the complex relation of four aforementioned straight lines. In our case, the complex relation of four points, obviously, is equal to the complex relation of the straight lines coinciding with vectors $x_{a}^{*}, x_{b}^{*}, x^{*}$ and $[x]^{*}$. Out of these four, the first two are also isotropic.

\section{CONCLUSIONS}

The concept representing a physical quantity as a vector of the pseudo-Euclidean plane means knowing the center of probability distribution and degree of uncertainty for this quantity. This possibility of natural and, in essence, necessary combination of the center of probability distribution and the degree of uncertainty for a quantity, is a reason to favor this geometrical concept of representing physical quantities.

The suggested approach allows to express a certain property of the physical object, that in particular may be expressed via a physical quantity, through a pseudo-Euclidean angle. This approach is a natural development of representing a physical quantity as a numerical axis.

Here comes the question: how exactly shall we use this representation in describing physical processes? In other words, what is a practical value of these results?

Since the combination of the center of distribution and dispersion around this center for a given quantity is defined by a two-dimensional vector, the equations of interest shall operate with these vectors instead of otherwise commonplace quantities. An example in the appendix illustrates such approach.

\section{APPENDIX}

\section{Example}

The sensitivity of an induction sensor to magnetic induction is described by the formula:

$S=\omega m A$,

where $\omega$ - cyclic frequency of induction varying, $w$ - number of windings in the coil, $A$ - projection of the coil's area on the plane, perpendicular to induction. Let $\omega$ lay in the range of $(5970 \div 6590) \mathrm{s}^{-1}$, with a probability of 0.95 ; let also $w=1293$. In the end, let us take it as given that $A$ lays in the range of $(1.22 \div 1.24) \cdot 10^{-3} \mathrm{~m}^{2}$ with 0.95 probability for different sensors of the same model. The formula for sensitivity in vector form will look as

\section{$\ln S=\ln \omega+\ln w+\ln A$.}

For the sake of simplicity, let us call: $\ln S=S^{*}, \ln \omega=\omega^{*}$, $\ln \boldsymbol{w}=w^{*}, \ln \boldsymbol{A}=A^{*}$. Then let's break each vector down into components, one of which is directed along the numerical axis and another - along the full vector of the quantity. The result of this transformation is

$\boldsymbol{a}_{0 S}+S^{*}=\boldsymbol{a}_{0 \boldsymbol{\omega}}+\omega^{*}+\boldsymbol{a}_{0 \boldsymbol{W}}+w^{*}+\boldsymbol{a}_{0 \boldsymbol{A}}+A^{*}$,

i.e. each quantity is represented by the sum of the distribution center and expanded uncertainty. It can be said that

$a_{0 S}=a_{0 \omega}+a_{0 w}+a_{0 A}$

is an algebraic sum of the logarithmic expected values, and

$S^{*}=\omega^{*}+w^{*}+A^{*}$

is a vector sum of the expanded uncertainties. (I) and (II) are, obviously, identical; however it is important to keep in mind that vectors in (I) are full vectors, whereas in (II) the same vectors become coordinate vectors, should the coordinate system change.

Let us determine the relative expanded uncertainty $\gamma_{s}$, taking into account that $w=$ const. According to the vector addition formula:

$\gamma_{S}=\left|S^{*}\right|=\sqrt{\left|\omega^{*}\right|^{2}+\left|A^{*}\right|^{2}+2 g_{\omega A}\left|\omega^{*}\right|\left|A^{*}\right|}$,

where $g_{\omega A}$ is the coordinate of the metric tensor of the model space. This tensor, according to [6], is determined by the distribution type for $\omega$ and $A$, coverage probability, and ratio of the uncertainties of $\omega$ and $A$. To find $\left|\omega^{*}\right|$ and $\left|A^{*}\right|$, see (11). Assuming normal distribution for $\omega$ and uniform for $A$, when aforementioned coverage probability and variables are present, following the value tables for the metric tensor developed by one of the authors in [6], we arrive at $g_{\omega A} \cong 0.05$. As a result, it turns out that $\left|S^{*}\right|=0.051$.

Here is where methodology from [7] becomes handy. As it follows from earlier stated values for $\omega$ and $A$, their relative 
expanded uncertainties are 0.05 and 0.01 , respectively. Matching standard uncertainties at coverage factors of 1.96 and 1.65 are found to be 0.026 and $6.1 \cdot 10^{-3}$ respectively. The standard uncertainty of $S$ therefore can be found as $\sqrt{0.026^{2}+\left(6.1 \cdot 10^{-3}\right)^{2}}=0.027$. The assumption of a normal distribution for $S$ will produce $\gamma_{S}=0.027 \cdot 1.96=$ 0.053 , that in all practicality equals the result, obtained earlier. It is important to notice that we made a supposition regarding the type of resulting distribution.

Applying the Monte Carlo method does not require such supposition; however, it requires a considerable increase of computing power. In this case, when statistical sampling of 10,000 values is exercised, $\gamma_{S}=0.052$, i.e. a comparable result yet again.

\section{REFERENCES}

[1] Tarbeyev Y.V., "Development of fundamental research in the area of theoretical metrology", 3-d All-State conference on theoretical metrology: presentation theses, Leningrad, 1986, pages 4-7 (in Russian)

[2] "Encyclopedia of Mathematics in five volumes", vol. 1, Moscow, 1977, 1152 col. (in Russian)

[3] Shutz, B., "Geometrical methods in Mathematical Physics", Translation from English, Moscow, 1984, 303 p, (in Russian)

[4] Mazin V.D., "Description of properties of physical objects in piecewise Euclidean and piecewise Riemann spaces", Scientific and technical bulletin of SPBSTU (Computing, measuring and operating systems), Petersburg, 1993 (in Russian)

[5] Novitsky P.V., Zograf I.A., "Evaluation of errors estimation in measurement results", Second edition, Leningrad, 1991, 303 p. (In Russ.)

[6] Mazin V.D., Chepushtanov A.N., "Properties and application technology of a vector-analytical method for an estimation of uncertainty in measurement", 5 International Conference on Precision Measurement, Ilmenau, 2008.

[7] "Guide to the Expression of Uncertainty in Measurement", JCGM 2008. 